Die Grenzen der Selbststeuerung von Unternehmen

\title{
Neue Rolle für den Staat
}

\section{Staatliche Kontrolle von Unternehmen sollte bei erfolgreicher Corporate Gover- nance zunehmend überflüssig werden. Die enttäuschenden Erfahrungen mit ein- zelnen Instrumenten sowie verstärkte regulative Maßnahmen von Regierungs- seite weisen aber deutlich auf die Grenzen der Idee der normativ geregelten Eigensteuerung hin. Für staatliche Regelsetzung zum Schutz der Umwelt gibt es - aus guten Gründen - immer noch Notwendigkeiten.}

Von Christian Hey

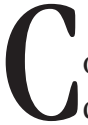
orporate Governance zielt in einer modernen Auslegung auch auf die nachhaltige Selbststeuerung von Unternehmen (1). Hierzu gehören Standards für Berichte, Managementsysteme oder den Dialog mit der Öffentlichkeit, sowie Selbstverpflichtungen mit Handlungszielen. Die Hoffnung der Anhänger einer Corporate Governance ist, dass, je leistungsfähiger diese Systeme der Selbststeuerung hinsichtlich persistenter Umweltprobleme werden, desto verzichtbarer auch die staatliche Kontrolle wird. Im Idealfall substituiert eine nachhaltige Corporate Governance die direkte staatliche Steuerung.

Dennoch findet dieser Substitutionsprozess nur schleichend und mit Rückschlägen statt. Im Klimaschutz, der Abfall- und der Chemikalienpolitik werden Selbstverpflichtungen gerade durch Instrumente der Regelsteuerung abgelöst. Die letzte Legislaturperiode war in Deutschland die regulativ aktivste in der Geschichte der deutschen Umweltpolitik. Auch Analysen der europäischen Umweltpolitik zeigen, dass die sogenannten neuen Instrumente der Umweltpolitik noch weit vom Durchbruch entfernt sind, auch wenn die derzeitige Rhetorik der Europäischen Kommission diesen bald erwarten lässt (2).

Handelt es sich bei diesen Trends um ein letztes Aufbäumen von staatsfixierten ewig Gestrigen oder deutet sich an, dass die Substitutionshoffnungen der Corporate Governance überzogen und unrealistisch waren?

\section{Grenzen der Eigenverantwortung}

Eigenverantwortliches Handeln wird in der umweltpolitischen Diskussion regelmäßig als Alternative zur staatlichen Regulierung angesehen. Zweifelsohne gibt es zahlreiche Einzelbeispiele von Pionierunternehmen, die auch unter den ge- gebenen ökonomischen Rahmenbedingungen vorzeigbare Umweltleistungen freiwillig erbringen, sei es durch die Entwicklung besonders umweltfreundlicher Produkte oder durch neue Produktionsverfahren. Neben strategischen Entscheidungen des Managements und historisch gewachsenen Produkt- und Produktionskonzepten, sind oft drei Faktoren ausschlaggebend für eine solche Pionierrolle. Entweder besetzen Unternehmen Marktnischen für einen umweltbewussten Verbraucherkreis, oder sie nehmen präventiv eine unternehmenspolitisch bedrohliche Skandalisierung durch Umweltgruppen oder ihre Kunden vorweg, oder sie versuchen, staatliche Regulierung durch frühzeitige Anpassung zu vermeiden (3).

Die ersten beiden Erfolgsbedingungen unternehmerischen Pionierverhaltens sind in vielen Einzelfällen gegeben, nicht aber als Regel zu erwarten. Zumindest solange nicht, wie die Marktsignale und das Steuer- und Subventionssystem umweltschädliche Verhaltensweisen insgesamt honorieren (4). Viele Selbstverpflichtungen der Wirtschaft stehen alleine schon deshalb unter dem Verdacht, nicht wesentlich mehr als „Business as Usual“

\begin{tabular}{|} 
Inserieren Sie \\
in Ökologisches Wirtschaften! \\
Sprechen Sie genau Ihre Zielgruppe an. Gesamt- \\
auflage von 1.500 Heften. Erscheint 5 x jährlich. \\
Fordern Sie noch heute die Mediadaten an! \\
Frau Dagmar Huber berät Sie gerne. \\
Kontakt: \\
Dagmar Huber, ökom Verlag \\
Waltherstr. 29,80337 München \\
E-Mail: huber@oekom.de
\end{tabular}

anzubieten, weil kein Mechanismus einer verbandsinternen Lasten- und Verantwortungszuweisung besteht. Ohne einen solchen Mechanismus ist aber ein Umweltziel jenseits des ohnehin zu Erwartenden verbandsintern kaum durchsetzbar. Ambitioniertere Selbstverpflichtungen setzen in der Regel den Willen und die glaubwürdige Drohung des Staates voraus, im Falle des Scheiterns auch auf die traditionelle Regelsteuerung zurückzugreifen. Der „stick behind the door" war eine wichtige Erfolgsbedingung von Selbstverpflichtungen. Insgesamt gibt auch die neueste Evaluation der OECD zur umweltpolitischen Effektivität freiwilliger Ansätze Anlass zur Ernüchterung (5).

\section{Leistungsgrenzen des Staates}

Die Diskussion um die Leistungsgrenzen des Regierens hat eine lange Tradition., Je nach theoretischem Blickwinkel werden die Informationsprobleme einer problemadäquaten Feinsteuerung, die begrenzte Resonanzfähigkeit und -willigkeit der Wirtschaft, die internen Koordinationsprobleme des sektoral und vertikal fragmentierten Staates oder der Einflussverlust des Nationalstaates in einer entgrenzten und globalisierten Wirtschaft thematisiert.

Bereits in der theoretischen Diskussion ziehen die Steuerungsskeptiker daraus aber nicht die Schlussfolgerung, der Staat müsse sich generell aus der Verantwortung zurückziehen. Vielmehr suchen sie nach intelligenten Verkoppelungen zwischen dem hierarchisch steuernden Staat und einer die Autonomie respektierenden Eigenverantwortung. ,Kontextsteuerung“ und „Supervision", die Verkoppelung von Selbststeuerung und staatlicher Steuerung oder der Einsatz von prozeduralen Instrumenten der Umweltpolitik, die reflexive Lernprozesse auslösen, sind einige der Empfehlungen eines „kooperativen Regierens“ mit Verbänden, die die oben genannten Steuerungsprobleme zu lösen versuchen (6). Der Staat bleibt in diesen Modellen wichtiger Impulsgeber und Moderator. Er verteilt Einflusschancen, setzt Spielregeln und ökonomische Anreize, verzichtet aber darauf, ein bestimmtes Ergebnis festzulegen oder ein bestimmtes Handeln vorzuschreiben. Solche Formen der indirekten Steuerung sind jedoch an bestimmte situative Bedingungen gekoppelt. Der Staat als ,Supervisor“", der tätig wird, wenn er dazu eingeladen wird, kann dies nur in bestimmten günstigen Konstellationen realisieren. Dazu zählen ähnliche Ziele bei Staat und Unternehmen, die sie alleine aber nicht erreichen können. In der Forschungs- und Technologiepolitik 
finden sich viele Konstellationen für die Produktion solcher ,kollateralen Güter“. In der Umweltpolitik sind sie weniger häufig und meist weniger harmonisch.

Dies gilt auch für die Verkoppelung von Selbststeuerung und Hierarchie in der Normung, wie sie zur Zeit insbesondere im Kontext einer Integrierten Produktpolitik auf europäischer Ebene angestrebt wird. Bisher nicht erreichte Voraussetzung für eine erfolgreiche Verkoppelung sind die Herstellung eines künstlichen Pluralismus in den Gremien zwischen den Vertretern von Schutzinteressen und denen von Produktionsinteressen, ein Mindestmaß an öffentlicher Transparenz, die Bereitschaft des Staates, als ,gleichberechtigter" Vertreter in diesen Gremien öffentliche Interessen wahrzunehmen, die Bildung mehrheitsfähiger Koalitionen, die mehr als die Standardisierung des Status Quo wollen, sowie die Fähigkeit des Staates, unbefriedigende Normungsergebnisse zu sanktionieren. Weiche, kooperative Steuerungsmodelle erfordern zumeist eine erhebliche und faktisch selten gegebene Bereitschaft, Kapazitäten und Ressourcen für deren Funktionieren zur Verfügung zu stellen. Auch die in den 90er Jahren verfolgte politische Strategie einer Kontextsteuerung durch die Korrektur des Preissystems mit Hilfe einer ökologischen Steuerreform stößt auf Akzeptanzgrenzen, die die Attraktivität des Instruments in letzter Zeit vermindert haben.

\section{Die unterschätzte Leistungs- fähigkeit des Staates}

Bemerkenswert sind jedoch, gerade in der Umweltpolitik, die Strategien des Staates, auf die oben beschriebenen Strukturprobleme staatlicher Steuerung zu reagieren, ohne auf das Instrumentarium der Steuerung über allgemein verbindliche Regeln zu verzichten. Dies soll exemplarisch verdeutlicht werden:

- Das Informationsproblem: Auch im Rahmen einer traditionellen Regelsteuerung konnten informationsgenerierende Prozesse erzeugt werden, die zum Teil sogar das Problem eines strategischen Understatements technologischer Potenziale verhütet haben. Im Rahmen der IVURichtlinie, die die Genehmigung und die Emissionskontrolle von Industrieanlagen regelt, wurde zum Beispiel ein Informationsaustausch zwischen Unternehmen, Genehmigungsbehörden und Umweltverbänden organisiert, durch den für zahlreiche Industriebranchen der Stand der Technik in einer integrierten Gesamtschau do- kumentiert wurde (7). In diesem und in anderen Fällen schafft die staatliche Umweltpolitik Anreize dafür, dass Unternehmen Informationen bereitstellen, insbesondere um eine Übersteuerung zu verhindern. Illusorisch wäre natürlich der Anspruch, die Informationsprobleme einer Integrierten Produktpolitik ganzheitlich regeln zu wollen. Die Komplexität einer ganzheitlichen Umweltverbesserung von Hundertausenden von Produkten hinsichtlich all ihrer Umweltaspekte ist kaum zu bewältigen. Machbar ist dagegen ein problemfokussiertes Vorgehen bei besonders relevanten Produkten. Die umweltpolitische Regulierung des Autos ist hierfür ein relativ erfolgreiches Beispiel.

- Das „Resonanzproblem“: In wichtigen Teilbereichen, insbesondere der Luftreinhaltepolitik und dem Gewässerschutz, hat die traditionelle Umweltpolitik beachtliche Erfolge vorzuweisen. Wenn technische Möglichkeiten existieren, scheint das Resonanzproblem in der Umweltpolitik nicht allzu gravierend zu sein. Schwieriger ist es allerdings, Sektordynamiken zu steuern, wie das Verkehrswachstum oder die Abfallmengen beziehungsweise die dort beobachtbaren Ausweichreaktionen der Abfallströme in niedrig regulierte Bereiche. Hier versagt die traditionelle Regelsteuerung - viel ist aber auch nicht von einer Corporate Governance zu erwarten. Am ehesten greifen hier verschiedene Strategien der Umweltpolitikintegration.

- Das Koordinationsproblem und Internationalisierungsproblem: Der Nationalstaat hat sich sowohl gegenüber den Bundesländern als auch hinsichtlich der europäischen und internationalen Kooperation erstaunliche Gestaltungsspielräume erhalten können. Politische Blockaden auf der internationalen Ebene konnten zum Teil durch nationale Vorreiterrollen umgangen werden, die international diffundierten. Die Blockaden, die die deutsche Variante eines verflochtenen und parteipolitisch überlagerten Föderalismus auch in der Umweltpolitik erzeugt, konnten durch Europäisierung der Umweltpolitik unterlaufen werden (8). Zwischen den politischen Ebenen haben sich stabile, informelle und formale Politiknetzwerke herausgebildet, die beachtliche kooperative Leistungen hervorbringen. Gerade in einem komplexen Mehrebenensystem fällt damit dem Nationalstaat mit seinen spezifischen Ressourcen eine besondere Rolle als zentraler, strategiefähiger und verhandelnder Akteur zu. Diese Rolle kann ihm niemand abnehmen.

\section{Fazit: Umweltschutz braucht Stautlichkeit}

Vor dem Hintergrund der hier skizzierten Analyse ist es verfrüht, Todesgesänge auf den Nationalstaat und die traditionelle Regelsteuerung in der Umweltpolitik anzustimmen. Die Regelsteuerung, insbesondere wenn sie durch kooperative Elemente modernisiert wird und die Chancen der Verkoppelung verschiedener Steuerungsmuster nutzt, hat in vieler Hinsicht entscheidende Leistungsvorteile gegenüber der auf Eigenverantwortung setzenden Corporate Governance oder Selbstverpflichtungen der Wirtschaft. Es ist durchaus sinnvoll, dass entgegen dem Trend der theoretischen Diskussion, die deutsche und die europäische Umweltpolitik in der Praxis die Chancen der Regelsteuerung weiter genutzt haben.

\section{Anmerkungen}

(1) Die in diesem Artikel vertretene Meinung ist die persönliche Auffassung des Autors und stellt keine Ratsmeinung dar.

(2) Knill, C./ Lenschow, A.: Modes of Regulation in the Governance of the European Union: towards a Comprehensive Evaluation. In: European Integration online Papers Vol. 7, 2003. Download unter: http://eiop.or.at/eiop/ texte/2003-001a.htm

(3) Jakob, K.: Innovationsorientierte Chemikalienpolitik. Politische, soziale und ökonomische Faktoren des verminderten Gebrauchs geführlicher Stoffe, München, 1999.

(4) Rat von Sachverständigen für Umweltfragen (Hg.): Umweltgutachten 2002. Für eine neue Vorreiterrolle, Stuttgart, 2002.

(5) OECD (Ed.): Voluntary Approaches for Environmental Policy: Effectiveness, Efficiency and Usage in Policy Mixes, Paris 2003.

(6) Heinelt, H./ Athanassopoulou, E./ Getimis, P. et al.: Prozedurale Umweltpolitik der EU. Umweltverträglichkeitsprüfungen und Öko-Audits im Ländervergleich, Opladen 2000.

(7) UBA (Ed.): The Sevilla process: A driver for environmental performance in industry, Proceedings of the European Conference, 6-7 April 2000, UBA Texte 16/00, Berlin 2000.

(8) Eichener, V.: Das Entscheidungssystem der Europäischen Union. Instiutionelle Analyse und demokratietheoretische Bewertung, Opladen 2000.

\section{Der Autor}

Dr. Christian Hey ist Generalsekretär des Rates von Sachverständigen für Umweltfragen (SRU).

Kontakt: SRU, Reichpietschufer 60, 10785 Berlin, Tel. 263696-110, E-Mail: christian.hey@uba.de 
(c) 20I0 Authors; licensee IÖW and oekom verlag. This is an article distributed under the terms of the Creative Commons Attribution Non-Commercial No Derivates License (http://creativecommons.org/licenses/by-nc-nd/3.o/), which permits unrestricted use, distribution, and reproduction in any medium, provided the original work is properly cited. 